\title{
Can postsurgical hypocortisolism be predicted presurgically?
}

Precautionary steroid substitutive therapy is indicated in all patients with adrenal incidentaloma who undergo unilateral adrenalectomy, regardless of normal cortisol secretion before surgery.

Postsurgical hypocortisolism occurs in all patients after removal of an adrenal mass causing overt cortisol excess (Cushing syndrome). Whether postsurgical hypocortisolism occurs in patients who undergo surgical removal of an adrenal incidentaloma, which causes only a subtle cortisol hypersecretion (subclinical hypercortisolism), was unknown. The group of Iacopo Chiodini (University of Milan, Italy) decided to investigate whether the biochemical parameters of cortisol secretion before surgery could predict the development of postsurgical hypocortisolism.

The researchers enrolled 60 patients who underwent surgical removal of an adrenal incidentaloma. Hypothalamicpituitary-adrenal (HPA) axis function was assessed by a low-dose corticotropin stimulation test and an insulin tolerance test if indicated, 2 months after surgery.

The study showed "no parameters or combination of parameters of HPA axis activity have enough diagnostic accuracy to reliably exclude the possibility of postsurgical hypocortisolism." Chiodini concludes further, "if the appearance of postsurgical hypocortisolism is considered indicative of the presence of subclinical hypercortisolism before surgery, these results confirm that the diagnosis of subclinical hypercortisolism, as performed to date, is probably not sensitive enough."

Linda Koch

Original article Eller-Vainicher, C. et al. Post-surgical hypocortisolism after removal of an adrenal incidentaloma: is it predictable by an accurate endocrinological work-up before surgery? Eur. J. Endocrinol. doi:10.1530/ EJE-09-0775 